\title{
RGS4 causes increased mortality and reduced cardiac hypertrophy in response to pressure overload
}

\author{
Jason H. Rogers, ${ }^{1,2}$ Praveen Tamirisa, ${ }^{1,2}$ Attila Kovacs, ${ }^{1}$ Carla Weinheimer, ${ }^{1}$ \\ Michael Courtois, ${ }^{1}$ Kendall J. Blumer, ${ }^{2}$ Daniel P. Kelly, ${ }^{1}$ and Anthony J. Muslin ${ }^{1,2}$ \\ ${ }^{1}$ Center for Cardiovascular Research, Department of Medicine, and \\ ${ }^{2}$ Department of Cell Biology and Physiology, Washington University School of Medicine, St. Louis, Missouri 63110, USA
}

Address correspondence to: Anthony J. Muslin, Center for Cardiovascular Research, Box 8086,

Washington University School of Medicine, 660 S. Euclid Avenue, St. Louis, Missouri 63110, USA.

Phone: (314) 747-3525; Fax: (314) 362-0186; E-mail: amuslin@imgate.wustl.edu.

Received for publication March 4, 1999, and accepted in revised form July 27, 1999.

RGS family members are GTPase-activating proteins (GAPs) for heterotrimeric G proteins. There is evidence that altered RGS gene expression may contribute to the pathogenesis of cardiac hypertrophy and failure. We investigated the ability of RGS4 to modulate cardiac physiology using a transgenic mouse model. Overexpression of RGS4 in postnatal ventricular tissue did not affect cardiac morphology or basal cardiac function, but markedly compromised the ability of the heart to adapt to transverse aortic constriction (TAC). In contrast to wild-type mice, the transgenic animals developed significantly reduced ventricular hypertrophy in response to pressure overload and also did not exhibit induction of the cardiac "fetal" gene program. TAC of the transgenic mice caused a rapid decompensation in most animals characterized by left ventricular dilatation, depressed systolic function, and increased postoperative mortality when compared with nontransgenic littermates. These results implicate RGS proteins as a crucial component of the signaling pathway involved in both the cardiac response to acute ventricular pressure overload and the cardiac hypertrophic program.

J. Clin. Invest. 104:567-576 (1999).

\section{Introduction}

Postnatal mammalian cardiomyocytes respond to mechanical stress and growth factor action by undergoing a hypertrophic response (1). This response is characterized by an increase in cell size, protein synthesis, and organization of contractile proteins into sarcomeres (2) and by an induction of specific genes, including atrial natriuretic factor (ANF) (3), the immediate early proto-oncogene c-fos (4), and myosin light chain-2 (MLC-2) (5). Cardiomyocyte hypertrophy leads to growth of the entire heart, resulting in thickening of the ventricular walls with an attendant reduction in wall stress. The clinical consequences of human cardiac hypertrophy are very significant and include the development of serious cardiac arrhythmias, of diastolic dysfunction that can result in pulmonary edema and fluid overload, and of congestive heart failure $(6,7)$. Cardiac hypertrophy is not always associated with a poor prognosis. For example, the development of cardiac hypertrophy in professional athletes does not predict a poor outcome, and heart size decreases when exercise levels are reduced $(8,9)$. This finding and others have led investigators to hypothesize that hypertrophy may be a necessary adaptation to increased environmental stress and that hypertrophy becomes maladaptive only in its latter stages.

In cultured cardiomyocytes, mechanical stress or ligands such as phenylephrine (10), endothelin-1 (11), and angiotensin II (12) promote a hypertrophic response.
These 3 agonists signal through heterotrimeric G proteins: endothelin-1 (13) and angiotensin II (14) bind to 7-transmembrane receptors that are coupled to $\mathrm{G}_{\mathrm{q}}$ proteins, whereas phenylephrine binds to $\alpha_{1}$-adrenergic receptors that are coupled to $G_{i}$ and $G_{q}$ proteins (15). Previous work has demonstrated that mechanical stress may lead to the local release of angiotensin II or endothelin-1 in the heart (16). Heterotrimeric G proteins consist of $\alpha, \beta$, and $\gamma$ subunits that form a complex in unstimulated cells (17-19). These proteins are activated by 7 -transmembrane receptors. With agonist stimulation, guanine nucleotide exchange occurs on the $\alpha$ subunit, resulting in the binding of GTP to the $\alpha$ subunit that leads to the dissociation of $\beta \gamma$ dimers. In contrast, GTPase activity causes GTP to dissociate from the $\alpha$ subunit, leading to the reformation of heterotrimers.

The ability of $G$ proteins to cause cardiac hypertrophy and failure has recently been examined in transgenic mouse model systems (20-22). Four-fold overexpression of $G_{\alpha q}$ in cardiac tissue resulted in increased heart weight and cardiomyocyte size, as well as in a dramatic increase in the expression of the ANF and $\beta$-myosin heavy chain ( $\beta$-MHC) genes (20). Furthermore, echocardiographic imaging in transgenic mice revealed impaired contractility with $G_{\alpha q}$ overexpression, an altered Starling relationship, and reduced contractile response to dobutamine stimulation. At higher levels of $G_{\alpha q}$ overexpression, biventricular failure and death occurred in several animals (20). In another 
Table 1

In vivo echocardiographic assessment, tight TAC

\begin{tabular}{lccc}
\hline \multicolumn{3}{c}{ Baseline } & Tight TAC \\
& NTG & $5 x$-RGS4 & $5 x$-RGS4 \\
& $(n=6)$ & $(n=6)$ & $(n=3)$ \\
& $625 \pm 44$ & $599 \pm 64$ & $670 \pm 23$ \\
HR (beats per min $)^{\mathrm{A}}$ & $3.24 \pm 0.13$ & $3.28 \pm 0.17$ & $3.88 \pm 0.10^{\mathrm{B}}$ \\
LVIDd $(\mathrm{mm})$ & $1.58 \pm 0.20$ & $1.51 \pm 0.10$ & $3.01 \pm 0.10^{\mathrm{B}}$ \\
LVIDs $(\mathrm{mm})$ & $0.62 \pm 0.08$ & $0.62 \pm 0.03$ & $0.42 \pm 0.07^{\mathrm{B}}$ \\
PWd $(\mathrm{mm})$ & $0.69 \pm 0.10$ & $0.68 \pm 0.05$ & $0.48 \pm 0.02^{\mathrm{B}}$ \\
IVSd $(\mathrm{mm})$ & $62.7 \pm 9.4$ & $62.9 \pm 5.0$ & $53.5 \pm 7.2$ \\
LVM $(\mathrm{mg})^{\mathrm{A}}$ & $0.40 \pm 0.07$ & $0.40 \pm 0.03$ & $0.23 \pm 0.02^{\mathrm{B}}$ \\
RWT & $51 \pm 5$ & $54 \pm 4$ & $22 \pm 1^{\mathrm{B}}$ \\
FS $(\%)$ & & &
\end{tabular}

Echocardiographic measurements obtained from transthoracic M-mode tracings of 5x RGS4-myc and transgenic littermates (NTG) at baseline and 5xRGS4-myc mice 1-2 days after tight TAC. HR, heart rate; LVIDd and LVIDs, end-diastolic and end-systolic LV internal dimensions, respectively; PWd and IVSd, end-diastolic posterior wall and intraventricular septal thickness, respectively; LVM, M-mode echocardiogram-derived LV mass; RWT, relative wall thickness (PWd+IVSd/LVIDd); FS, fractional shortening (LVIDd-LVIDs/LVIDd). ${ }^{A} P=$ NS between all groups. ${ }^{B} P<0.05$ vs. baseline groups.

study, transient expression of a constitutively active mutant form of $\mathrm{G}_{\alpha \mathrm{q}}$ in postnatal heart tissue resulted in the development of cardiac hypertrophy, dilatation, and death at between 8 and 30 weeks of age (21). In a third study, cardiac-specific expression of an inhibitor of $\mathrm{G}_{\mathrm{q}}$-mediated signaling blocked the induction of cardiac hypertrophy in response to pressure overload (22).

Activated 7-transmembrane receptors catalyze the formation of $\mathrm{G}_{\alpha}$-GTP complexes, which in turn regulate the activity of effector molecules. The rate at which GTP is hydrolyzed determines the strength and duration of 7-transmembrane receptor-generated signals. $G_{\alpha}$ subunits have weak intrinsic GTP hydrolysis activity $\left(\mathrm{k}_{\mathrm{CAT}}=2-5^{-1}\right)$, as do small $\mathrm{G}$ proteins, such as ras, which hydrolyze GTP much more slowly than $G_{\alpha}$ (23). GTPase-activating proteins (GAPs) are present in cells to promote the deactivation of small $\mathrm{G}$ proteins. For example, p120GAP accelerates the intrinsic GTPase activity of ras by 100,000 -fold (23). Recently, GAPs for heterotrimeric $G$ proteins were identified and were named RGS proteins (regulators of $G$ protein signaling) $(24,25)$. RGS proteins bind with high affinity to GDP$\mathrm{AlF}_{4}{ }^{-}$complexes of $\mathrm{G}_{\alpha}$ subunits that mimic the putative pentavalent transition state, and RGS proteins stimulate GTP hydrolysis catalytically by at least 50 -fold over the basal rate (27-29). The higher affinity of RGS proteins for the GDP- $\mathrm{AlF}_{4}^{-}$complex of $\mathrm{G}_{\alpha}$ than for the GTPY-S-bound form suggests that RGS proteins act by stabilizing the transition state between the GTP and GDP-bound forms (23). Biochemical studies performed in vitro using purified proteins have demonstrated that RGS1, RGS4, RGS10, and RGS16 (RGS-r) have GAP activity toward $\alpha$ subunits of heterotrimeric $G$ proteins of the $G_{i}$ and $G_{q}$, but not $G_{s}$ families (26-29).

We have demonstrated previously that RGS3 and RGS4 mRNAs are expressed in the heart (30), and other investigators have shown recently that several additional RGS family members, including RGS1, RGS5, and RGS6, are expressed in ventricular tissue (31). The expression pattern of RGS family members in cardiac tissue is altered in pathophysiologic states and in response to cardiomyocyte dissociation $(30,31)$. We have hypothesized that alterations in RGS gene expression may affect $G$ protein-mediated signal transduction in the heart. To address this possibility, we found previously that overexpression of RGS4 in cultured cardiomyocytes inhibits phenylephrine- and endothelin-induced cardiomyocyte hypertrophy (32). To evaluate whether RGS4 overexpression could inhibit cardiac hypertrophy in response to physiological stimuli in live animals, we have generated a transgenic mouse model system.

\section{Methods}

Transgenic mouse generation. The coding region of the rat RGS4 cDNA with a 3'-triple-myc-1-epitope tag was subcloned into a vector (clone 26; gift of Jeffrey Robbins, University of Cincinnati, Cincinnati Ohio, USA) containing the $\alpha$-myosin heavy chain ( $\alpha$-MHC) promoter and an SV-40 polyadenylation site (33). Linearized DNA was injected into the pronuclei of 1cell C57BL/ $6 \times$ SJL embryos at the National Institute of Child Health and Human Development Transgenic Mouse Development Facility at the University of Alabama-Birmingham (Birmingham, Alabama, USA) as described previously (34). Progeny were analyzed by PCR to detect transgene integration. Two founders were obtained and dot blot analysis confirmed that 5 copies of the transgene were incorporated into 1 line (5x-RGS4-myc), while 8 copies of the transgene were incorporated into the second line (8x-RGS4-myc).

All research involving the use of mice was performed in strict accordance with protocols approved by the Animal Studies Committee of Washington University.

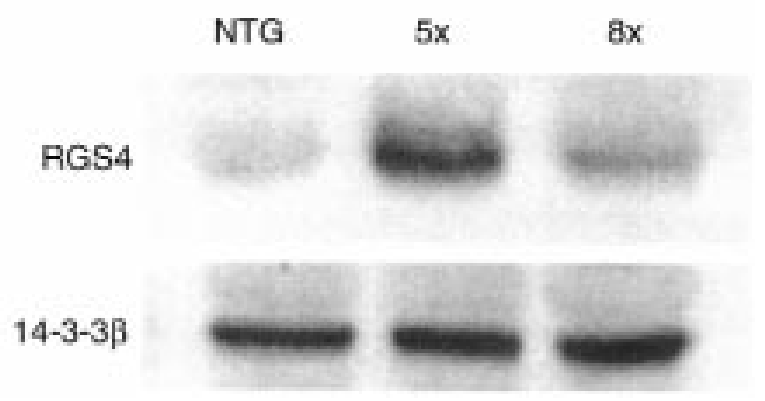

Figure 1

Characteristics of RGS4-myc cardiac tissue. Increased ventricular RGS4 protein levels in 5x-RGS4-myc (5x) and 8x-RGS4-myc (8x) mice compared with nontransgenic littermate mice (NTG). The 5x-RGS4myc mice express 4 - to 5 -fold excess protein, whereas the $8 x$-RGS4myc mice express only 2 - to 3 -fold excess protein. A hamster monoclonal anti-RGS4 antibody and a rabbit polyclonal anti-14-3-3 $\beta$ antibody (to confirm equal loading) were used. Similar results were obtained in 6 hearts in each group. 


\begin{tabular}{lcccc} 
& $\begin{array}{c}\text { Total } \\
\text { "tight" } \\
\text { TAC }\end{array}$ & $\begin{array}{c}\mathbf{1} \text { day } \\
\text { survival }\end{array}$ & $\begin{array}{c}\mathbf{3} \text { day } \\
\text { survival }\end{array}$ & $\begin{array}{c}\mathbf{7} \text { day } \\
\text { survival }\end{array}$ \\
\hline $\begin{array}{c}\text { NTG littermates } \\
\text { (of 5x-RGS4-myc mice) } \\
\text { NTG C57BL } \times \text { SJL }\end{array}$ & 9 & $8(89 \%)$ & $7(78 \%)$ & $7(78 \%)$ \\
5x-RGS4-myc & 48 & $41(85 \%)$ & $30(63 \%)$ & $30(63 \%)$ \\
& 18 & $6(33 \%)$ & $2(11 \%)$ & $2(11 \%)$ \\
$\mathbf{8 x}$-RGS4-myc & 14 & $2(14 \%)$ & $0(0 \%)$ & $0(0 \%)$ \\
\hline
\end{tabular}

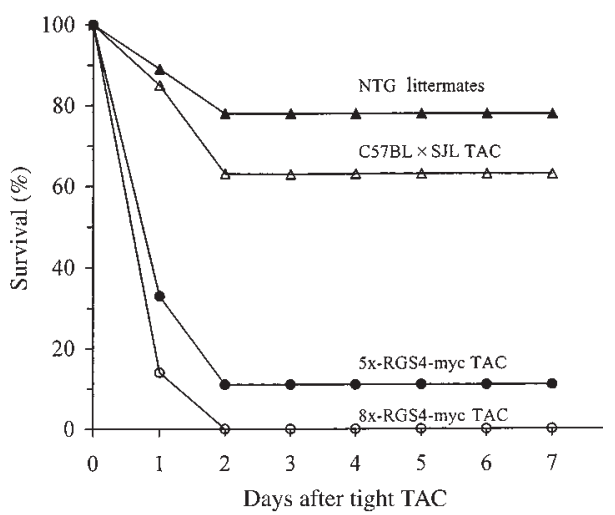

\section{Figure 2}

Decreased survival of RGS4-myc transgenic mice after tight TAC. Survival rates after tight TAC in 8x-RGS4-myc mice, 5x-RGS4-myc mice, nontransgenic littermates of 5x-RGS4-myc mice (NTG littermates), and nontransgenic congenic mice (NTG C57BL $\times$ SJL TAC).

Protein analysis. Ventricular tissue cytosolic extracts were used to analyze levels of RGS4-myc protein by immunoblotting as described previously (30). Murine monoclonal anti-myc-1 epitope (Oncogene Research Products, Cambridge, Massachusetts, USA), hamster monoclonal anti-RGS4, and rabbit polyclonal anti-14-3-3 $\beta$ (Santa Cruz Biotechnology Inc., Santa Cruz, California, USA) antibodies were employed. Horseradish peroxidase-conjugated goat anti-mouse, goat anti-hamster, or goat anti-rabbit polyclonal antibodies (ICN Pharmaceuticals Inc., Costa Mesa, California, USA) were used. Bands were viewed using the enhanced chemiluminescence (ECL) system (Amersham Pharmacia Biotech, Piscataway, New Jersey, USA). Densitometric analysis of immunoblots using NIH Image software revealed that $5 \mathrm{x}$-RGS-myc mice contained 4- to 5-fold excess RGS4 protein, and that $8 \mathrm{x}-\mathrm{RGS} 4-\mathrm{myc}$ mice contained 2- to 3-fold excess RGS4 protein.

Mitogen-activated protein kinase activity assays. Intracardiac injection of phenylephrine was performed as described previously (22). After 90 seconds, ventricular tissue was quickly isolated and snap frozen in liquid nitrogen (30). Cytosolic extracts of ventricular tissue were separated by SDS-PAGE, and proteins were electrophoretically transferred to nitrocellulose filters. Filters were blocked in Tris-buffered saline containing $1 \%$ Tween-20 (TBS/T) and 30\% BSA. Filters were washed and incubated with a 1:1,000 dilution of anti-active ERK-1 mitogen-activated protein (MAP) kinase murine mAb (Promega Corp., Madison, Wisconsin, USA). Filters were extensively washed in TBS/T, then were incubated with horseradish peroxidase-conjugated anti-mouse secondary antibody (Amersham Pharmacia Biotech). Bands were viewed using the ECL system
(Amersham Pharmacia Biotech) and analyzed by densitometry as described above.

Transverse aortic constriction. Transverse aortic constriction (TAC) was performed largely as described previously (35-37). In brief, 12-week-old mice were anesthetized with a mixture of xylazine $(16 \mathrm{mg} / \mathrm{kg})$ and ketamine $(80 \mathrm{mg} / \mathrm{kg})$. The chest was opened, and following blunt dissection through the intercostal muscles, the thoracic aorta was identified. A 7-0 silk suture was placed around the transverse aorta and tied around a 26-gauge blunt needle ("tight" TAC) (37) or a 25gauge blunt needle ("loose" TAC), which was subsequently removed. The chest was closed with a pursestring suture. At the end of the procedure, the incision was closed in 2 layers with an interrupted suture pattern. The mice were kept on a heating pad until responsive to stimuli. The surgeon was blinded to the transgenic status of the mice. Sham-operated animals
Table 2

In vivo echocardiographic assesment, loose TAC

\begin{tabular}{lcccc}
\hline & \multicolumn{2}{c}{ Sham } & \multicolumn{2}{c}{ Loose TAC } \\
& NTG & $5 x$-RGS4 & NTG & $5 x-$ RGS4 \\
& $(n=5)$ & $(n=8)$ & $(n=4)$ & $(n=4)$ \\
HR (beats per min) $)^{\mathrm{A}}$ & $549 \pm 32$ & $626 \pm 40$ & $560 \pm 52$ & $642 \pm 29$ \\
LVIDd $(\mathrm{mm})^{\mathrm{A}}$ & $3.50 \pm 0.14$ & $3.35 \pm 0.16$ & $3.35 \pm 0.31$ & $3.42 \pm 0.10$ \\
LVIDs $(\mathrm{mm})^{\mathrm{A}}$ & $2.01 \pm 0.24$ & $1.70 \pm 0.19$ & $1.59 \pm 0.27$ & $1.73 \pm 0.14$ \\
PWd $(\mathrm{mm})$ & $0.54 \pm 0.04$ & $0.66 \pm 0.03$ & $1.07 \pm 0.11^{\mathrm{B}}$ & $0.68 \pm 0.04^{\mathrm{C}}$ \\
IVSd $(\mathrm{mm})$ & $0.61 \pm 0.02$ & $0.69 \pm 0.03$ & $1.16 \pm 0.11^{\mathrm{B}}$ & $0.75 \pm 0.08^{\mathrm{C}}$ \\
LVM $(\mathrm{mg})$ & $60.4 \pm 5.1$ & $68.9 \pm 5.5$ & $147 \pm 29.0^{\mathrm{B}}$ & $78.6 \pm 12.6^{\mathrm{D}}$ \\
RWT & $0.33 \pm 0.02$ & $0.41 \pm 0.03$ & $0.68 \pm 0.08^{\mathrm{B}}$ & $0.42 \pm 0.03^{\mathrm{C}}$ \\
FS $(\%)^{\mathrm{A}}$ & $43 \pm 5$ & $50 \pm 3$ & $54 \pm 4$ & $50 \pm 3$ \\
BWgt $(\mathrm{g})^{\mathrm{A}}$ & $27.5 \pm 1.4$ & $27.8 \pm 0.9$ & $29.9 \pm 2.3$ & $27.5 \pm 0.3$ \\
SBP $(\mathrm{mmHg})$ & $131 \pm 6.4$ & $127 \pm 7.2$ & $195 \pm 19$ & $176 \pm 5^{\mathrm{E}}$ \\
LVMI $(\mathrm{mg} / \mathrm{g})$ & $3.06 \pm 0.10$ & $3.00 \pm 0.08$ & $4.24 \pm 0.33^{\mathrm{B}}$ & $3.53 \pm 0.27^{\mathrm{D}}$ \\
& & & &
\end{tabular}

Echocardiographic measurements obtained from transthoracic M-mode tracings of 5x-RGS4myc and nontransgenic littermates (NTG) at 1 week after loose TAC or sham operations. BWgt, preoperative body weight; SBP, systolic blood pressure in ascending aorta proximal to TAC site; LVMI, gravimetrically determined LV mass index (LV mass $/ B W g t) .{ }^{A} P=N S$ between all groups. ${ }^{B} P<0.001$ vs. sham groups. ${ }^{C} P<0.001$ vs. NTG loose TAC. $D P<0.05$ vs. NTG loose TAC. E $P=$ NS vs. NTG loose TAC. 


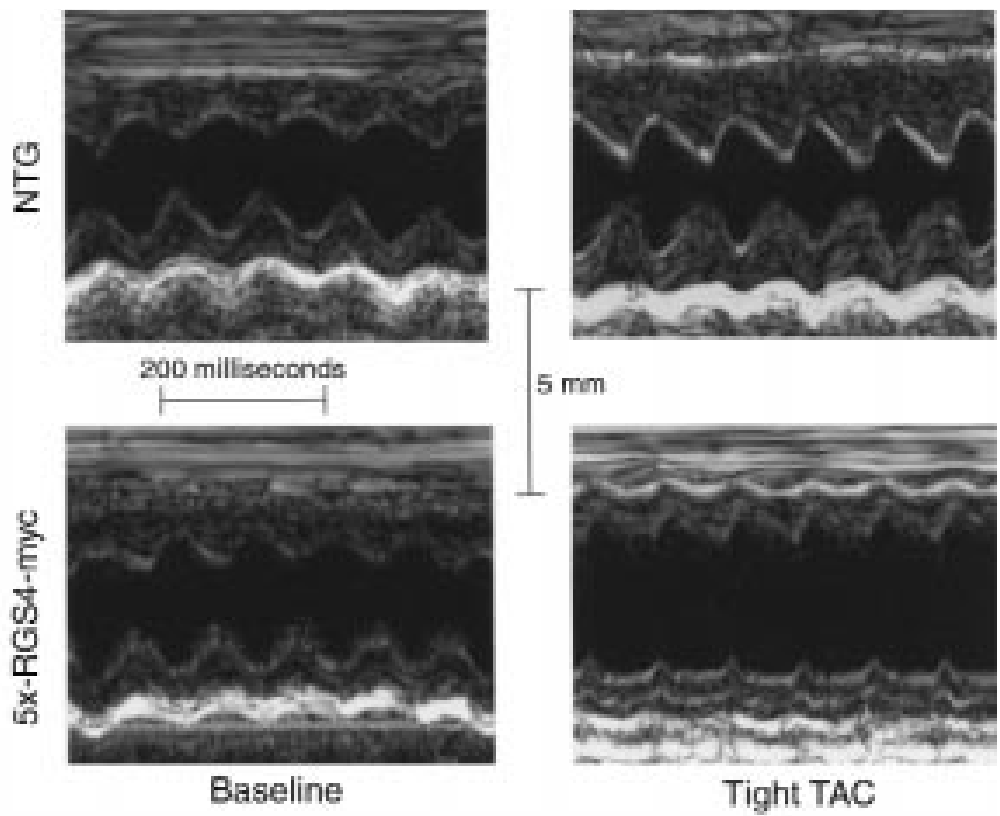

\section{Figure 3}

Analysis of cardiac function in 5x-RGS4-myc transgenic mice by M-mode echocardiography. Representative transthoracic M-mode echocardiographic tracings in a 5x-RGS4-myc mouse and a nontransgenic littermate (NTG) at baseline. TAC images shown for nontransgenic ( 1 week after tight TAC) and 5x-RGS4-myc (premorbid, 1 day after TAC) mice.

underwent the identical surgical procedure, except that the aortic constriction was not placed. After 7 days, surviving animals were sacrificed and hearts were dissected out and weighed.

Cardiac catheterization. Mice were anesthetized 7 days after TAC with a mixture of xylazine $(16 \mathrm{mg} / \mathrm{kg})$ and ketamine $(80 \mathrm{mg} / \mathrm{kg})$. Closed-chest cardiac catheterization was performed by identifying and cannulating the right carotid artery and advancing a 1.4F Millar catheter into the ascending aorta, proximal to the aortic constriction, where it was secured; then hemodynamic measurements were recorded.

Echocardiography. Transthoracic echocardiography was performed in anesthetized mice (intraperitoneal injection of $0.01 \mathrm{~mL}$ of $2.5 \%$ Avertin per gram of body weight) by using an Acuson Sequoia 256 Echocardiography System (Acuson Corp., Mountain View, California, USA) equipped with a $15-\mathrm{MHz}$ (15L8) transducer as described previously $(38,39)$. Premorbid mice were lethargic and did not require Avertin sedation.

Dobutamine stimulation and evaluation of response. Transthoracic echocardiography and hemodynamic measurements were performed as described previously (40).

Northern blotting. Total RNA was isolated from frozen murine ventricular tissue by the guanidinium thiocyanate and phenol method (RNA-STAT60; Tel-Test Inc., Friendswood, Texas, USA). RNA $(15 \mu \mathrm{g})$ was separated by $1 \%$ formaldehyde-agarose gel electrophoresis and transferred and cross-linked to nylon membranes. ANF, GAPDH, and medium chain acyl-CoA dehydrogenase (MCAD) probes were labeled with $\left[\alpha-{ }^{32} \mathrm{P}\right] \mathrm{dCTP}$ using random hexamers and the Klenow fragment of DNA polymerase I (Amersham Pharmacia Biotech). Blots were prehybridized, hybridized, and washed as described previously (30). Bands were viewed and analyzed using a Bio-Rad GS-525 Molecular Imager Sys- tem with Molecular Analyst 2.1.2 software (Bio-Rad Laboratories Inc., Hercules, California,USA).

Histologic analysis of ventricular tissue. Seven days after TAC, wild-type and 5x-RGS4-myc mice were sacrificed and ventricular tissue was obtained. Ventricular tissue was fixed in $4 \%$ paraformaldehyde in phosphatebuffered saline, embedded in paraffin, and sectioned using a microtome. Tissue sections were stained with Masson's trichrome.

Apoptosis assay. Evaluation of apoptosis was performed in situ by terminal deoxynucleotidyl transferase (TdT) assay using the FragEl kit (Oncogene Research Products, Cambridge, Massachusetts, USA).

Statistical analysis. All data are reported as mean \pm SEM. Statistical analysis was performed by 2 -tailed Student's $t$ test, $\chi^{2}$ analysis, and ANOVA, where applicable. Multiple group comparison was carried out by ANOVA with Fisher's post hoc comparison. A $P$ value less than 0.05 was considered to be statistically significant.

\section{Results}

Generation of RGS4-myc transgenic mice. We have shown previously that the RGS3 and RGS4 genes are expressed in the heart (30). We demonstrated recently that overexpression of RGS4 can inhibit the action of phenylephrine and endothelin-1, but not basic fibroblast growth factor, in cultured cardiomyocytes (32). To determine whether RGS4 could inhibit cardiac hypertrophy in an animal model system, we generated transgenic mice with a construct that contained the $\alpha$-MHC promoter that has been demonstrated previously to direct modest embryonic and increased postnatal ventricular gene transcription (33). The $\alpha$-MHC promoter was linked to the coding region of rat RGS4 that contained a 3'-triple-myc-1 epitope tag (RGS4-myc). Two founder mice were obtained and used to generate independent lines. In 1 line, there was integration of 5 
copies of the transgene ( $5 x-R G S 4-m y c)$, and in the second line there was integration of 8 copies of the transgene (8x-RGS4-myc).

Expression of the RGS4-myc fusion protein was analyzed in mice, using both an anti-RGS4 mAb and an anti-myc-1 epitope mAb (data not shown), revealing that ventricular tissue isolated from 5x-RGS4-myc mice contained 4- to 5-fold excess RGS4 protein and, despite a higher copy number, 8x-RGS4-myc mice contained only 2 - to 3 -fold excess RGS4 protein. The levels of a control protein, 14-3-3 $\beta$, were identical in 5x-RGS4-myc mice, $8 x$-RGS4-myc transgenic mice, and their nontransgenic littermates (Figure 1). All 5x-RGS4-myc and 8x-RGS4-myc heterozygote transgenic mice appeared grossly normal at birth and lived for at least 6 months in the absence of experimental intervention. Baseline echocardiographic parameters were identical in $5 \mathrm{x}-$ RGS4-myc mice and nontransgenic littermates (Table 1). The 8x-RGS4-myc mice also had normal left ventricular (LV) systolic function, chamber size, and wall thickness. The hearts of 5x-RGS4-myc and 8x-RGS4-myc mice appeared grossly normal. Histologic analysis of 5xRGS4-myc and 8x-RGS4-myc mouse ventricular tissue revealed normal cardiomyocyte appearance compared with nontransgenic littermates (data not shown).

Response of RGS4-myc mice to tight TAC. We next examined the ability of mice to develop cardiac hypertrophy in response to provocative stimulation by using tight TAC. In this procedure, a $60-70 \%$ stenosis in the transverse aorta is created by surgical ligation (35-37). In nontransgenic littermates, TAC was tolerated, and nearly $80 \%$ of animals survived for at least 1 week (Figure 2). After 1 week, most mice developed significant cardiac hypertrophy that was easily detected by echocardiographic study.

In contrast, 5x-RGS4-myc transgenic mice did not tolerate tight TAC, and most died within hours to 2 days after the procedure (Figure 2). Echocardiography of premorbid 5x-RGS4-myc animals within 1 to 2 days after tight TAC demonstrated ventricular dilatation, wall thinning, depressed cardiac function, and no appreciable increase in LV mass (Table 1 and Figure 3).

$\chi^{2}$ analysis revealed that the decrease in survival observed in 5x-RGS4-myc transgenic mice (11\%) 7 days after tight TAC was statistically significant when compared with either nontransgenic littermates $(78 \%)(P=$ $0.0001)$ or to nontransgenic congenic C57BL $\times$ SJL animals $(63 \%)(P=0.0001)$. To exclude the possibility that $5 x$-RGS4-myc transgenic mice were uniquely sensitive to anesthesia or thoracotomy, sham operations were performed that were identical to the TAC procedure, except that the aorta was not ligated after it was identified by dissection. All 6 sham-operated 5x-RGS4-myc mice tolerated the procedure and survived more than 7 days.

Tight TAC was performed on 8x-RGS4-myc mice, and they also did not tolerate TAC. All 8x-RGS4-myc mice (14:14) died within 3 days of tight TAC (Figure 2), and echocardiography on premorbid mice demonstrated findings identical to those found in premorbid 5x-RGS4-myc mice after tight TAC.

Response of RGS4-myc mice to loose TAC. Given the high mortality rate observed in 5x-RGS4-myc and 8x-RGS4myc mice following tight TAC, to increase postoperative survival we adopted a modified form of the TAC procedure where a less restrictive band was placed on the transverse aorta, resulting in a stenosis of approximately $40-50 \%$. Either this modified form of TAC (loose TAC) or a sham operation was performed on $5 \mathrm{x}$ RGS4-myc mice and also on nontransgenic mice. All mice were approximately 12 weeks of age and were matched by body weight (Table 2 ). Cardiac catheterization was performed on all surviving mice at 1 week after surgery to confirm that loose TAC resulted in a physiologically significant stenosis. Catheterization revealed that the mean ascending aortic systolic blood pressure (SBP) was $131 \pm 6 \mathrm{mmHg}$ in sham-operated nontransgenic animals and $195 \pm 19 \mathrm{mmHg} 7$ days after loose TAC. In 5x-RGS4-myc animals, the SBP was $127 \pm 7 \mathrm{mmHg}$ in the sham-operated group and $176 \pm$ $5 \mathrm{mmHg} 7$ days after loose TAC (Table 2). Mice were

\begin{tabular}{lcccc} 
& $\begin{array}{c}\text { Total } \\
\text { loose } \\
\text { TAC }\end{array}$ & $\begin{array}{c}\mathbf{1} \text { day } \\
\text { survival }\end{array}$ & $\begin{array}{c}\mathbf{3} \text { day } \\
\text { survival }\end{array}$ & $\begin{array}{c}\mathbf{7} \text { day } \\
\text { survival }\end{array}$ \\
\hline $\mathbf{5 x}$-RGS4-myc sham & 8 & $8(100 \%)$ & $\mathbf{8 ( 1 0 0 \% )}$ & $8(100 \%)$ \\
Nontransgenic C57BL x SJL & 20 & $19(95 \%)$ & $15(75 \%)$ & $15(75 \%)$ \\
$\mathbf{5 x - R G S 4 - m y c ~}$ & 18 & $7(39 \%)$ & $6(33 \%)$ & $6(33 \%)$ \\
\hline
\end{tabular}

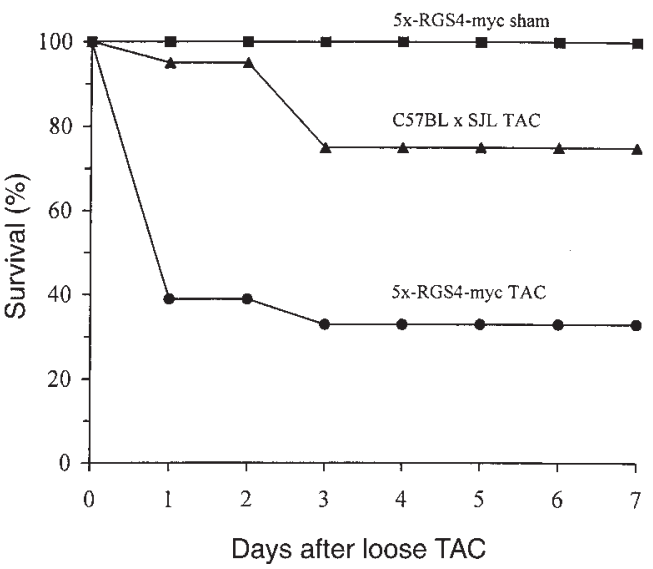

\section{Figure 4}

Decreased survival of RGS4-myc transgenic mice after loose TAC. Survival rates after loose TAC in 5x-RGS4-myc mice (5x-RGS4-myc TAC) and nontransgenic congenic C57BL $\times$ SJL mice $(C 57 B L \times S J L T A C)$ and after a sham operation in 5x-RGS4-myc mice $(5 x-R G S 4-m y c$ sham). 


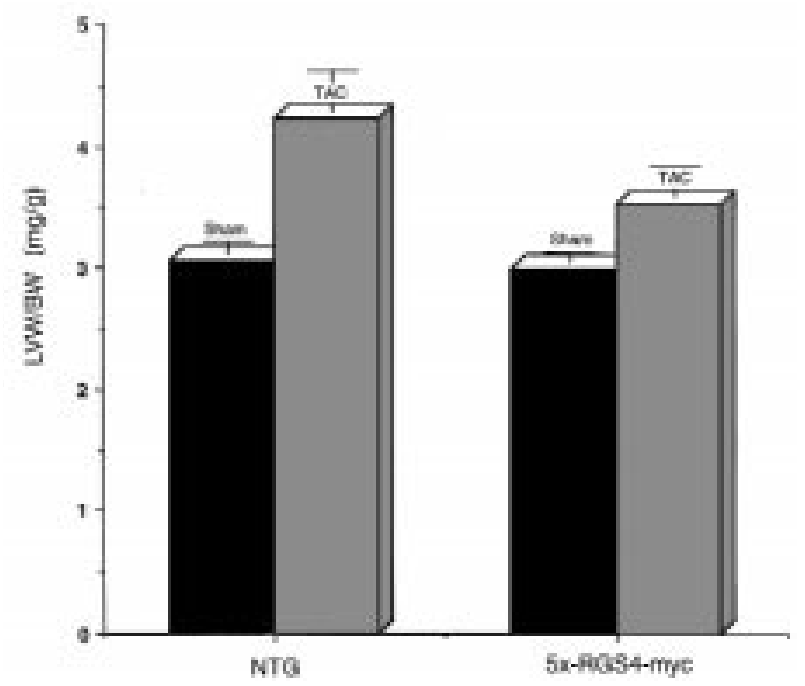

Figure 5

Reduced hypertrophic response to TAC in 5x-RGS4-myc mice. The $\mathrm{LV}$ weight/body weight ratio (LVW/BW), an index of LV mass, was determined 7 days after loose TAC or after a sham operation in nontransgenic congenic C57BL $\times$ SJL mice (NTG) or in 5x-RGS4-myc mice. Mice were excluded from LWW/BW analysis after TAC if the ascending aortic SBP was less than 2 SDs greater than the mean ascending aortic SBP obtained in sham-operated animals. The error bars represent the SEM.

excluded from analysis after loose TAC if the ascending aortic SBP was not at least 2 SDs greater than the mean ascending aortic SBP in sham-operated animals.

Seven days after loose TAC, nontransgenic mice had a survival rate of $75 \%$, and $5 x-$ RGS4-myc mice had an improved survival rate of $33 \%$, as compared with a $11 \%$ survival rate after tight TAC. The decrease in survival observed in 5x-RGS4-myc transgenic mice following loose TAC compared with nontransgenic mice at 7 days was statistically significant by $\chi^{2}$ analysis $(P$ $=0.0001)$ (Figure 4).

Echocardiographic assessment of 5x-RGS4-myc survivors 7 days after loose TAC revealed that they exhibited preserved LV function but had significantly less LV hypertrophy than nontransgenic survivors. Specifically, fractional shortening and LV end-diastolic and endsystolic dimensions were not statistically different in nontransgenic and 5x-RGS4-myc mice 1 week after loose TAC (Table 2). However, indices of LV hypertrophy such as end-diastolic posterior wall and intraventricular septal thickness, relative wall thickness, and Mmode derived LV mass were significantly less in $5 x$-RGS4-myc animals when compared with nontransgenic mice (Table 2). LV mass/body weight ratios (LVMI) were determined gravimetrically. The LVMI was $3.06 \pm 0.10 \mathrm{mg} / \mathrm{g}$ in sham-operated nontransgenic mice $(n=5)$ and $4.24 \pm 0.33 \mathrm{mg} / \mathrm{g}$ in nontransgenic banded mice 7 days after loose TAC $(n=4 ; P=0.0002)$. In the loose TAC group, the LVMI in nontransgenic animals increased by $39 \%$ compared with that in sham-operated mice (Table 2 and Figure 5). In contrast, the LVMI in $5 x$-RGS4-myc animals increased by only $18 \%$ after loose
TAC compared with sham-operated 5x-RGS4-myc mice. The LVMI for sham-operated 5x-RGS4-myc mice was $3.00 \pm 0.08 \mathrm{mg} / \mathrm{g}(n=8)$ and was $3.53 \pm 0.27 \mathrm{mg} / \mathrm{g}$ in 5x-RGS4-myc mice $(n=4) 7$ days after loose TAC $(P$ $=0.04)$ (Table 2 and Figure 5). The increase in LVMI observed in 5x-RGS4-myc mice (18\%) was significantly smaller than that observed in nontransgenic mice (39\%) after loose TAC $(P=0.02)$ despite similar mean ascending aortic SPBs.

Histologic analysis of LV tissue of 5x-RGS4-myc and nontransgenic littermates was performed on survivors 7 days after TAC. Nontransgenic animals exhibited typical myofibrillar disarray and fibrosis after TAC, whereas transgenic animals had little or no fibrosis and relatively preserved myofibrillar architecture (Figure 6).

Examination of the cardiac bypertrophy gene regulatory program in RGS4-myc mice after loose TAC. In wild-type mice, previous work has established that provocative stimulation by TAC induces the expression of "embryonic" genes such as ANF and MLC-2 (35) and reduces the expression of genes involved in the mitochondrial fatty acid oxidation pathway, such as MCAD (41). Loose TAC was performed on 5x-RGS4-myc mice and nontransgenic C57BL/ $6 \times$ SJL mice, and ventricular

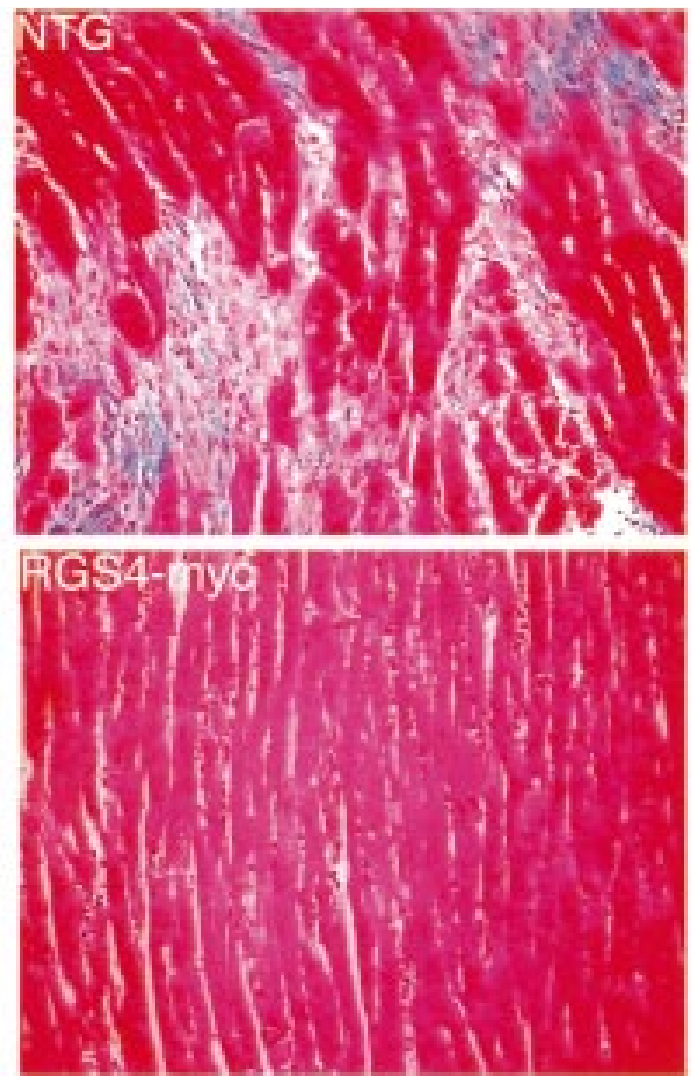

\section{Figure 6}

Histologic analysis of cardiac morphology after TAC. Ventricular tissue sections from 5x-RGS4-myc (RGS4-myc) and nontransgenic littermates (NTG) of 5x-RGS4-myc mice were stained with Masson's trichrome 1 week after loose TAC (original magnification $\times 200$ ). Note the increased extracellular matrix content (blue color), cardiomyocyte enlargement, and disarray in the nontransgenic cardiac tissue. 

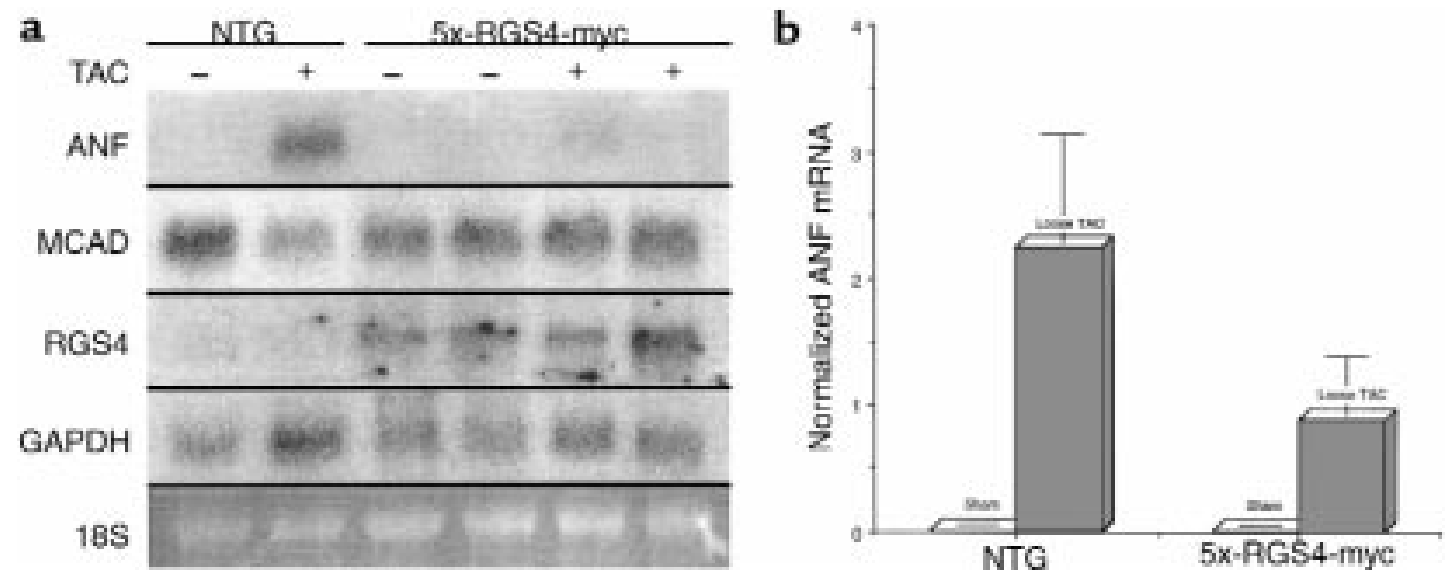

\section{Figure 7}

Northern blot analysis of cardiac gene expression after TAC. (a) Northern blot analysis of ANF, MCAD, RGS4, and GAPDH gene expression after loose TAC. Loose TAC (+) or a sham operation (-) was performed on 5x-RGS4-myc or nontransgenic (NTG) congenic C57BL $\times$ SJL mice. (b) Quantitative analysis of cardiac ANF gene expression 7 days after loose TAC. The relative intensities of the resultant bands were quantified in their linear range by automated 2-dimensional computer densitometry. The graph depicts normalized ANF mRNA levels in ventricular tissue obtained from 5x-RGS4-myc or nontransgenic congenic C57BL $\times$ SJL mice (NTG). RGS4 mRNA levels were normalized by GAPDH mRNA. Data are presented in arbitrary units and error bars reflect the SE of 4 determinations.

tissue was collected 7 days after the procedure. Northern blot analysis of RNA obtained from ventricular tissue revealed that loose TAC induced a marked increase in ANF expression in nontransgenic, but not 5x-RGS4-myc, animals (Figure 7a). Normalized ANF levels were significantly reduced in 5x-RGS4-myc mice when compared with nontransgenic animals after TAC (Figure $7 \mathrm{~b})(P=0.03)$. Loose TAC caused a marked decrease in MCAD expression in nontransgenic, but not $5 \mathrm{x}$-RGS4-myc, mice. Loose TAC did not affect RGS4-myc mRNA levels in 5x-RGS4-myc animals (Figure 7a).

Response of RGS4-myc mice to $\alpha$-adrenergic and $\beta$-adrenergic stimulation. To determine whether the ability of RGS4-myc ventricular tissue to respond to ligands that activate $G_{i}$ or $G_{q}$ proteins was impaired, hearts were infused with the $\alpha$-adrenergic ligand phenylephrine, and intracellular MAP kinase activation was evaluated. Previous work has demonstrated that intraventricular injection of phenylephrine results in a rapid $(90 \mathrm{sec}-$ onds) increase in cardiac MAP kinase activity (22). Nontransgenic littermates, but not 5x-RGS4-myc transgenic mice, exhibited increased MAP kinase activity in response to intracardiac phenylephrine infusion as measured by anti-active MAP kinase (ERK-1) immunoblotting (Figure 8).

To determine whether $5 \mathrm{x}$-RGS4-myc transgenic mice could respond normally to ligands that activate $\mathrm{G}_{\mathrm{s}}$ proteins in contrast to phenylephrine, which activates $G_{i}$ and $G_{q}$ proteins, we performed in vivo echocardiography and hemodynamic evaluations after graded infusions of the $\beta$-adrenergic agonist dobutamine $(40,42)$. Augmentation of LV contractility, assessed by peak LV $+\mathrm{dP} / \mathrm{dt}$ and heart rate in 5x-RGS4-myc mice, was comparable to nontransgenic mice, and there was no statistically significant difference in either peak $\mathrm{LV}+\mathrm{dP} / \mathrm{dt}$ or heart rate at any level of dobutamine infusion (Figure 9). This is consistent with the inability of RGS4 to inactivate $\mathrm{G}_{\mathrm{s}}$ proteins.

Examination of apoptosis in 5x-RGS4-myc nonsurvivors and survivors after TAC. To investigate whether apoptotic mechanisms play a role in the acute lethality or reduced hypertrophy phenotypes after TAC, ventricular tissue was isolated from $5 \mathrm{x}$-RGS4-myc mice that expired less than 2 days after TAC or that survived 7 days after TAC. Ventricular tissue was also collected from nontransgenic mice that survived 7 days after TAC. Staining of nuclei with a TdT assay revealed a paucity of apoptotic nuclei, and there was no statistically significant difference in apoptotic indices of $5 \mathrm{x}-$ RGS4-myc mice that died in less than 2 days or that survived to 1 week after TAC, compared with nontransgenic survivors at 1 week after TAC. Specifically, apoptotic indices were as follows: nontransgenic mice at 1 week after loose TAC, $0.87 \pm 0.43 \%$; $5 \mathrm{x}$-RGS4-myc mice at 1 week after loose TAC, $1.1 \pm 0.59 \%$; $5 x$-RGS4myc mice that expired less than 24 hours after TAC, $1.43 \pm 0.87 \%$ (Figure 10).

\section{Discussion}

In human patients, cardiac hypertrophy arises as a consequence of increased afterload, increased intracardiac blood volume, genetic abnormalities in cardiac sarcomeric proteins, or inborn errors of metabolism (43). Common causes of increased afterload in humans include hypertension and aortic stenosis, and these conditions are often associated with cardiac hypertrophy. In this study, we used TAC in mice as an experimental method to acutely increase afterload and thereby provoke cardiac hypertrophy (35-37). Aortic constriction triggers a rapid compensatory response that leads to increased cardiac contractility within 


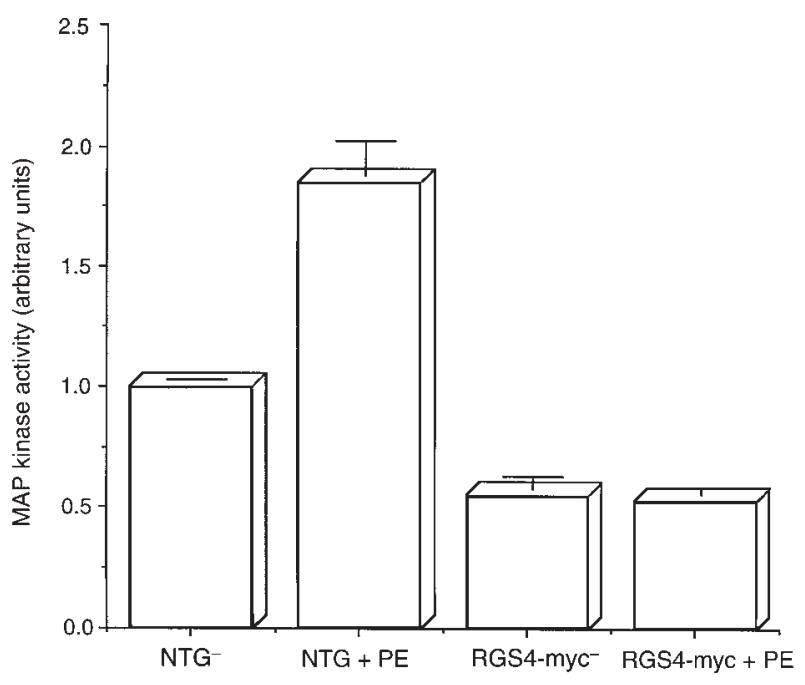

Figure 8

Reduced RGS4-myc cardiac response to the $G_{i} / G_{q}$-coupled ligand phenylephrine. The p44 MAP kinase activity was assessed in the ventricular tissue of RGS4-myc mice or their nontransgenic littermates 90 seconds after intracardiac infusion of phenylephrine (or control buffer). Immunoblots of cytosolic extracts were analyzed using an anti-active ERK-1 MAP kinase mAb. Equal amounts of total protein were loaded in each lane. Densitometric analysis of 3 separate experiments was performed using $\mathrm{NIH}$ Image software, and data are expressed as the mean signal intensity \pm SEM.

minutes to maintain cardiac output. If animals are unable to respond immediately to this increase in afterload, cardiac dilatation and hypokinesis ensue, often resulting in death. It has not been firmly established which ligands, if any, mediate this rapid response to increased cardiac afterload. Previous work has suggested that local intracardiac release of endothelin-1 or angiotensin II may be involved in this process, leading to $\mathrm{G}_{\mathrm{q}}$-protein activation (16). $\mathrm{G}_{\mathrm{q}}$ activation, in turn, could result in the activation of phospholipase $\mathrm{C}$, resulting in the generation of inositol triphosphate and the liberation of cytoplasmic calcium $(44,45)$. In addition, the immediate decrease in cardiac output following TAC is predicted to result in catecholamine release that would stimulate intracardiac $\alpha$-adrenergic and $\beta$-adrenergic receptors, stimulating $G_{i}$ protein and $G_{s}$ protein. In addition to the rapid inotropic response, there is a delayed increase in cardiomyocyte cell size resulting in cardiac hypertrophy that occurs over days to weeks. It is not known whether the stimuli that contribute to the rapid increase in contractility are also involved in promoting the delayed growth of cardiomyocytes.

The results described in this report suggest that RGS4 overexpression in mouse ventricular tissue inhibits the ability of the heart to compensate for an acute increase in afterload induced by TAC. Indeed, both 5x-RGS4-myc and 8x-RGS4-myc mice exhibited a marked increase in postoperative mortality following tight and loose TAC. Transgenic mice were examined by echocardiography before their demise, and this revealed LV dilatation with global hypokinesis. One interpretation of these results is that transgenic animals lacked the capacity to mount an inotropic response to increased afterload. However, inotropic and chronotropic response to the $\beta$-agonist dobutamine was preserved in 5x-RGS4-myc mice. An impaired $\beta$-adrenergic response does not appear to play a role in the acute mortality after TAC. In contrast to our results, when Akhter et al. targeted expression of a $G_{\alpha q}$ inhibitory peptide to the heart, postoperative mortality was not reported to increase after TAC when compared with nontransgenic animals, but TAC-induced cardiac hypertrophy was inhibited (22). One possible explanation for these differences is that RGS4 has the ability to inhibit $\mathrm{G}_{\mathrm{i}}$ and $\mathrm{G}_{\mathrm{q}}$, but the $\mathrm{G}_{\alpha \mathrm{q}}$ inhibitory peptide only inhibits $\mathrm{G}_{\mathrm{q}}$-mediated signaling.

We investigated whether myocyte apoptosis was responsible for the acute mortality following TAC, but found no increase in apoptotic indices in 5x-RGS4-myc animals following TAC. This is in contrast to a recent study that provides evidence that early lethality in pressure-overloaded hearts can occur through loss of the gp130-dependent myocyte survival pathway, resulting in myocyte apoptosis (46). An additional contrast is
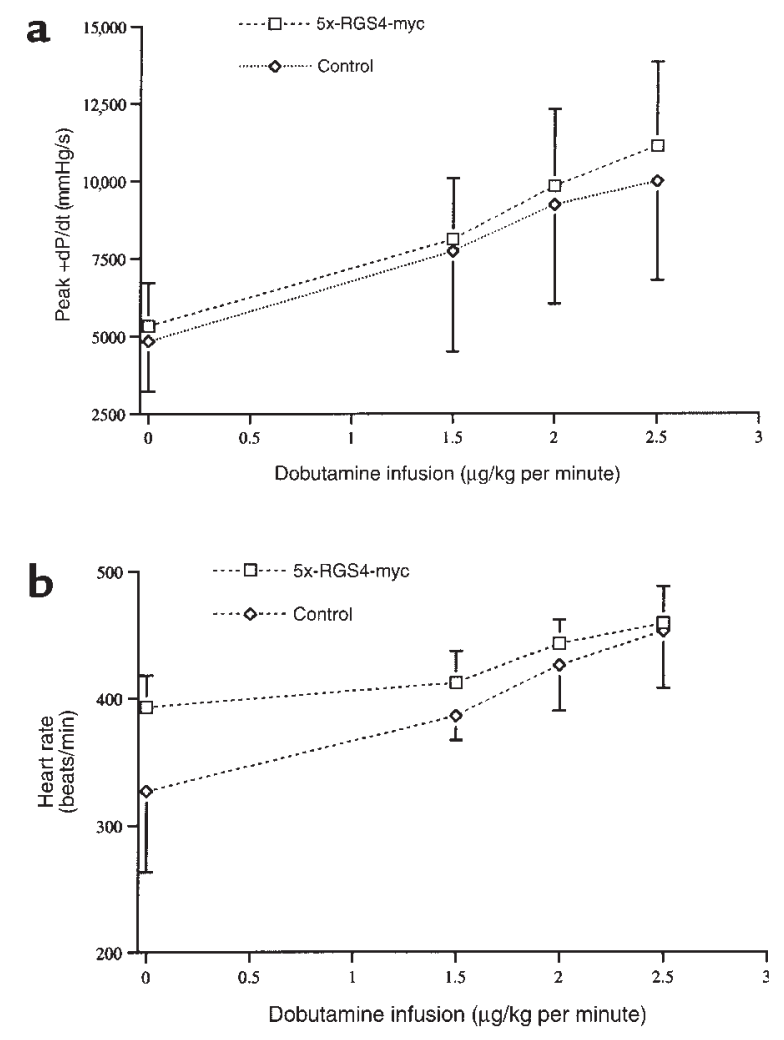

Figure 9

Preserved inotropic and chronotropic response of 5x-RGS4-myc mice to dobutamine. Peak LV $+\mathrm{dP} / \mathrm{dt}(\mathbf{a})$ and heart rate $(\mathbf{b})$ are shown at baseline and after progressive infusion of dobutamine in 5x-RGS4myc mice (squares; $n=3$ ) or nontransgenic C $57 \mathrm{BL} \times$ SJL mice (diamonds; $n=3)$. Peak $+d P / d t$, maximal first derivative of $L V$ pressure. $P=$ NS between $5 x-$ RGS4-myc mice and nontransgenic mice at any level of dobutamine infusion. 

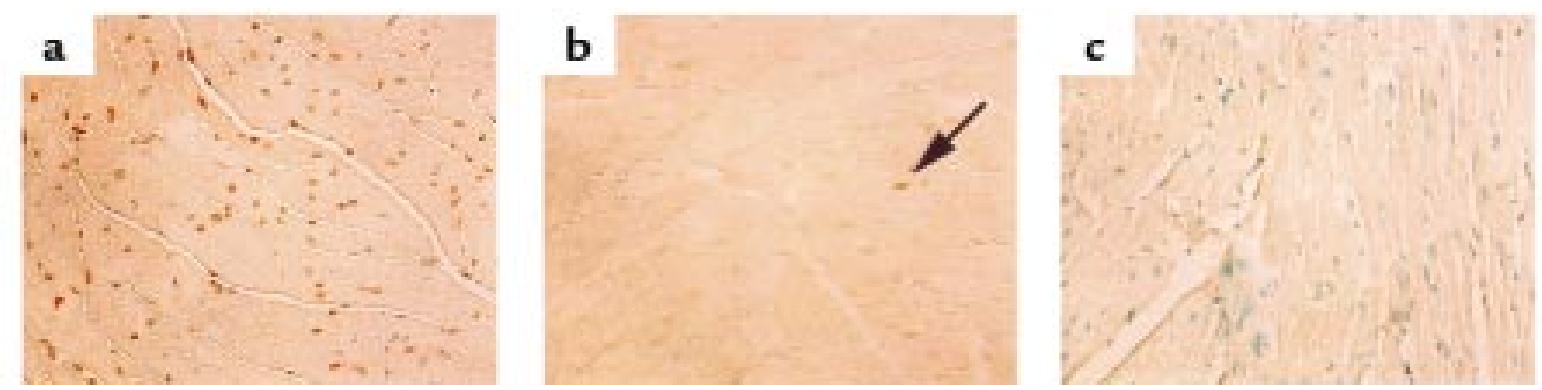

Figure 10

Apoptotic indices are not increased in 5x-RGS4-myc mice after TAC. All figures are of LV myocardium (original magnification $\times 400$ ), and are representative ofTdT assays performed. (a) Positive control: tissue incubated in DNase demonstrating pan staining of nucleic DNA by TdT-labeling assay. (b) 5x-RGS4-myc 1 week after loose TAC. Note paucity of apoptotic nuclei shown by the arrow. (c) 5x-RGS4-myc, death less than 24 hours after TAC. Note absence of apoptotic nuclei. Nonapoptotic nuclei are counterstained with methyl green.

that ventricular-specific gp130 knockout mice (gp130 $\mathrm{CKO}$ ) have normal induction of the embryonic gene program after TAC, whereas $5 x$-RGS4-myc mice have markedly attenuated induction of ANF. The $5 \mathrm{x}$-RGS4myc and gp130 CKO mice appear to represent distinct genetic modifiers of the biomechanical stress pathway in the heart.

In 5x-RGS4-myc transgenic animals that survived TAC, we observed that cardiac hypertrophy was blunted after 7 days, as measured by heart weight or induction of ANF, despite the presence of increased proximal aortic pressures. Animals that survived for 7 days had preserved LV function, and we do not know whether they would have lived for prolonged periods of time. In contrast, control animals that survived TAC developed profound cardiac hypertrophy after 7 days. An interesting question is whether transgenic animals that survived TAC would ultimately fare better than control banded animals. In other words, is hypertrophy beneficial to animals in response to TAC after the immediate postoperative period? Our experimental findings suggest that there is a biphasic response to increased afterload: an initial response that is characterized by increased contractility and a delayed hypertrophic response. Inhibition of the initial response has disastrous consequences that can result in death, but inhibition of the delayed response may not be disadvantageous. Experiments are underway to address these possibilities in greater detail.

In this work, we have used a transgenic approach that resulted in the overexpression of an RGS protein in the heart. This overexpression strategy is relevant to the response of the heart to increased afterload, as evidenced by our previous work demonstrating that pulmonary artery banding of nontransgenic mice leads to increased RGS4 expression in right ventricular tissue (30). Furthermore, recent work has demonstrated that RGS16 (RGS-r) is highly induced in cardiomyocytes shortly after dissociation from intact ventricular tissue (31). Taken together, these findings suggest that the heart may regulate the extent of the hypertrophic response by modulating RGS protein levels. RGS4 is a bonafide $G A P$ for $G_{i}$ and $G_{q}$ proteins, so a likely defect in the RGS4-myc mice is the inability of $\mathrm{G}_{i}$ or $\mathrm{G}_{\mathrm{q}}$ to transduce a signal that is required for both the acute and chronic responses to increased afterload.

\section{Acknowledgments}

We thank Ken Chien (University of California-San Diego, San Diego, California, USA) and Jeffrey Robbins (University of Cincinnati, Cincinnati, Ohio, USA) for reagents. We thank M. Hafeez Qureshi for technical assistance. We thank Mike Parmacek and Howard Rockman for technical advice. A.J. Muslin was supported by grants from the Barnes-Jewish Hospital Foundation, the American Heart Association, and the National Institutes of Health. K.J. Blumer and D.P. Kelly are Established Investigators of the American Heart Association. This work was done duing ther tenure of a post-doctoral fellowship from the American Heart Association, Heartland Affiliate Inc.

1. Sadoshima, J., and Izumo, S. 1997. The cellular and molecular response of cardiac myocytes to mechanical stress. Annu. Rev. Physiol. 59:551-571.

2. Thorburn, J., Frost, J.A., and Thorburn, A. 1994. Mitogen-activated protein kinases mediate changes in gene expression, but not cytoskeletal organization associated with cardiac muscle cell hypertrophy. J. Cell Biol. 126:1565-1572.

3. Knowlton, K.U., et al. 1991. Co-regulation of the atrial natriuretic factor and cardiac myosin light chain-2 genes during $\alpha$-adrenergic stimulation of neonatal rat ventricular cells. J. Biol. Chem. 266:7759-7768.

4. Izumo, S., Nadal-Ginard, B., and Mahdavi, V. 1988. Proto-oncogene induction and reprogramming of cardiac gene expression produced by pressure overload. Proc. Natl. Acad. Sci. USA. 85:339-343.

5. Lee, H., et al. 1988. $\alpha 1$-adrenergic stimulation of cardiac gene transcription in neonatal rat myocardial cells: effects on myosin light chain-2 gene expression. J. Biol. Chem. 263:7352-7358.

6. Levy, D., Garrison, R.J., Savage, D.D., Kannel, W.B., and Castelli, W.P. 1990. Prognostic implications of echocardiographically determined left ventricular mass in the Framingham Heart Study. N. Engl.J. Med. 322:1561-1566.

7. Messerli, F.H., and Soria, F. 1992. Hypertension, left ventricular hypertrophy, ventricular ectopy, and sudden death. Am. J. Med. 93:21S-26S.

8. Longhurst, J.C., and Stebbins, C.L. 1997. The power athlete. Cardiol. Clin. 15:413-429.

9. Shepard, R.J. 1996. The athlete's heart: is big beautiful? Br. J. Sports. Med. 30:5-10.

10. Simpson, P., McGrath, A., and Savion, S. 1982. Myocyte hypertrophy in neonatal rat cultures and its regulation by serum and by catecholamines. Circ. Res. 51:787-801.

11. Shubeita, H.E., et al. 1990. Endothelin induction of inositol phospholipid hydrolysis, sarcomere assembly, and cardiac gene expression in ventricular myocytes. J. Biol. Chem. 265:20555-20562.

12. Sadoshima, J., and Izumo, S. 1993. Molecular characterization of angiotensin II-induced hypertrophy of cardiac myocytes and hyperplasia of cardiac fibroblasts. Circ. Res. 73:413-423. 
13. Sokolovsky, M. 1993. Functional coupling between endothelin receptors and multiple G-proteins in rat heart myocytes. Receptors Channels. 1:295-304.

14. Wang, C., Jayadev, S., and Escobedo, J.A. 1995. Identification of a domain in the angiotensin II type 1 receptor determining Gq coupling by the use of receptor chimeras. J. Biol. Chem. 270:16677-16682.

15. Steinberg, S.F., Drugge, E.D., Bilezekian, J.P., and Robinson, R.B. 1985. Acquisition by innervated cardiac myocytes of a pertussis toxin-specific regulatory protein linked to the alpha 1-receptor. Science. 230:186-188.

16. Komuro, I., et al. 1995. Protein kinase cascade activated by mechanical stress in cardiocytes: possible involvement of angiotensin II. Eur. Heart J. 16(Suppl. C):8-11.

17. Gilman, A.G. 1987. G proteins: transducers of receptor-generated signals. Annu. Rev. Biochem. 56:615-649.

18. Hamm, H.E., and Gilchrist, A. 1996. Heterotrimeric G proteins. Curr. Opin. Cell Biol. 8:189-196.

19. Neer, E.J. 1995. Heterotrimeric G proteins: organizers of transmembrane signals. Cell. 80:249-257.

20. D'Angelo, D.D., et al. 1997. Transgenic G $\alpha$ q overexpression induces cardiac contractile failure in mice. Proc. Natl. Acad. Sci. USA 94:8121-8126.

21. Mende, U., et al. 1998. Transient cardiac expression of constitutively active $\mathrm{G} \alpha \mathrm{q}$ leads to hypertrophy and dilated cardiomyopathy by calcineurin-dependent and independent pathways. Proc. Natl. Acad. Sci. USA. 95:13893-13898

22. Akhter, S.A., et al. 1998. Targeting the receptor-Gq interface to inhibit in vivo pressure overload hypertrophy. Science. 280:574-577.

23. Tesmer, J.J.G., Berman, D.M., Gilman, A.G., and Sprang, S.R. 1997. Structure of RGS4 bound to $\mathrm{AlF}_{4}^{-}$-activated $\mathrm{G}_{i \alpha 1}$ : stabilization of the transition state for GTP hydrolysis. Cell. 89:251-261.

24. Druey, K.M., Blumer, K.J., Kang, V.H., and Kehrl, J.H. 1996. Inhibition of a $G$ protein-mediated MAP kinase activation by a new mammalian gene family. Nature. 379:742-746.

25. Koelle, M.R., and Horvitz, H.R. 1996. EGL-10 regulates G protein signaling in the C. elegans nervous system and shares a conserved domain with many mammalian proteins. Cell. 84:115-125.

26. Berman, D.M., Wilkie, T.M., and Gilman, A.G. 1996. GAIP and RGS4 are GTPase-activating proteins for the Gi subfamily of $G$ protein $\alpha$ subunits. Cell. 86:445-452.

27. Watson, N., Linder, M.E., Druey, K.M., Kehrl, J.H., and Blumer, K.J. 1996. RGS family members: GTPase activating proteins for $\alpha$ subunits of heterotrimeric G proteins. Nature. 382:172-175.

28. Huang, C., Hepler, J.R., Gilman, A.G., and Mumby, S.M. 1997. Attenuation of Gi- and Gq-mediated signaling by expression of RGS4 or GAIP in mammalian cells. Proc. Natl. Acad. Sci. USA. 94:6159-6163.

29. Natochin, M., Lipkin, V.M., and Artemyev, N.O. 1997. Interaction of human retinal RGS with G-protein alpha-subunits. FEBS Lett. 411:179-182.

30. Zhang, S., et al. 1998. RGS3 and RGS4 are G protein inhibitors in the heart. J. Mol. Cell. Cardiol. 30:269-276.
31. Kardestuncer, T., Wu, H., Lim, A.L., and Neer, E.J. 1998. Cardiac myocytes express mRNA for ten RGS proteins: changes in RGS mRNA expression in ventricular myocytes and cultured atria. FEBS Lett. 43:285-288.

32. Tamirisa, P., Blumer, K.J., and Muslin, A.J. 1999. RGS4 inhibits G protein signaling in cardiomyocytes. Circulation. 99:441-447.

33. Subramaniam, A., et al. 1991. Tissue-specific regulation of the alphamyosin heavy chain gene promoter in transgenic mice. J. Biol. Chem. 1266:24613-24620

34. Disch, D.L., et al. 1996. Transcriptional control of a nuclear gene encoding a mitochondrial fatty acid oxidation enzyme in transgenic mice: role for nuclear receptors in cardiac and brown adipose expression. Mol. Cell. Biol. 16:4043-4051.

35. Rockman, H.A., et al. 1991. Segregation of atrial-specific and inducible expression of an atrial natriuretic factor transgene in an in vivo murine model of cardiac hypertrophy. Proc. Natl. Acad. Sci. USA. 88:8277-8281.

36. Dorn, G.W., II, Robbins, J., Ball, N., and Walsh, R.A. 1994. Myosin heavy chain regulation and myocyte contractile depression after left ventricular hypertrophy in aortic-banded mice. Am. J. Physiol. 267:H400-H405.

37. Sakata, Y., Hoit, B.D., Liggett, S.B., Walsh, R.A., and Dorn, G.W., II. 1998. Decompensation of pressure-overload hypertrophy in Gaq-overexpressing mice. Circulation. 97:1488-1495.

38. Tanaka, N., et al. 1996. Transthoracic echocardiography in models of cardiac disease in the mouse. Circulation. 94:1109-1117.

39. Rockman, H.A., et al. 1998. Expression of a beta-adrenergic receptor kinase 1 inhibitor prevents the development of myocardial failure in gene-targeted mice. Proc. Natl. Acad. Sci. USA. 95:7000-7005.

40. Arber, S., et al. 1997. MLP-deficient mice exhibit a disruption of cytoarchitectural organization, dilated cardiomyopathy, and heart failure. Cell. 88:393-403

41. Sack, M.N., et al. 1996. Fatty acid oxidation enzyme gene expression is downregulated in the failing heart. Circulation. 94:2837-2842.

42. Nabauer, M., et al. 1988. Positive inotropic effects in isolated ventricular myocardium from non-failing and terminally failing human hearts. Eur. J. Clin. Invest. 18:600-606.

43. Jacob, R., Brandle, M., Dierberger, B., and Rupp, H. 1991. Functional consequences of cardiac hypertrophy and dilatation. Basic Res. Cardiol. 86(Suppl. 1):113-130

44. Smrcka, A.V., Hepler, J.R., Brown, K.O., and Sternweis, P.C. 1991. Regulation of polyphosphoinositide-specific phospholipase $\mathrm{C}$ activity by purified Gq. Science. 251:804-807.

45. Taylor, S.J., Chae, H.Z., Rhee, S.G., and Exton, J.H. 1991. Activation of the beta 1 isozyme of phospholipase $\mathrm{C}$ by alpha subunits of the $\mathrm{Gq}$ class of G proteins. Nature. 350:516-518.

46. Hirota, H., et al. 1999. Loss of a gp130 cardiac muscle cell survival pathway is a critical event in the onset of heart failure during biomechanical stress. Cell. 97:189-198. 